\title{
Long-chain acyl-CoA synthetase 2 is involved in seed oil production in Brassica
}

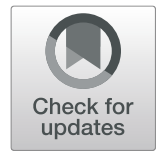
napus

\author{
Li-Na Ding ${ }^{\dagger}$, Shou-Lai Gu ${ }^{\dagger}$, Fu-Ge Zhu, Zhong-Yan Ma, Juan Li, Ming Li, Zheng Wang and Xiao-Li Tan
}

\begin{abstract}
Background: Triacylglycerols (TAGs) are the main composition of plant seed oil. Long-chain acyl-coenzyme A synthetases (LACSs) catalyze the synthesis of long-chain acyl-coenzyme A, which is one of the primary substrates for TAG synthesis. In Arabidopsis, the LACS gene family contains nine members, among which LACS1 and LACS9 have overlapping functions in TAG biosynthesis. However, functional characterization of LACS proteins in rapeseed have been rarely reported.
\end{abstract}

Results: An orthologue of the Arabidopsis LACS2 gene (BnLACS2) that is highly expressed in developing seeds was identified in rapeseed (Brassica napus). The BnLACS2-GFP fusion protein was mainly localized to the endoplasmic reticulum, where TAG biosynthesis occurs. Interestingly, overexpression of the BnLACS2 gene resulted in significantly higher oil contents in transgenic rapeseed plants compared to wild type, while BnLACS2-RNAi transgenic rapeseed plants had decreased oil contents. Furthermore, quantitative real-time PCR expression data revealed that the expression of several genes involved in glycolysis, as well as fatty acid (FA) and lipid biosynthesis, was also affected in transgenic plants.

Conclusions: A long chain acyl-CoA synthetase, BnLACS2, located in the endoplasmic reticulum was identified in $B$. napus. Overexpression of BnLACS2 in yeast and rapeseed could increase oil content, while BnLACS2-RNAi transgenic rapeseed plants exhibited decreased oil content. Furthermore, BnLACS2 transcription increased the expression of genes involved in glycolysis, and FA and lipid synthesis in developing seeds. These results suggested that BnLACS2 is an important factor for seed oil production in B. napus.

Keywords: BnLACS2, Oil contents, Glycolysis, Fatty acids, Lipid, Rapeseed (Brassica napus)

\section{Background}

Oilseed rape (Brassica napus. L) is one of the four major oil plants in the world, and plays an important role in producing vegetable proteins and edible oils for human consumption. Moreover, seed oils are important raw materials for biofuels and in the pharmaceutical industry with substantial economic value and high demand in recent years $[1,2]$. Thus, increasing the oil content of seeds is important for genetic breeders and has become a major topic of oil crop research $[3,4]$.

Vegetable oil is mainly accumulated during the seed maturation phase and supplies carbon and energy for

\footnotetext{
* Correspondence: xltan@ujs.edu.cn

† Li-Na Ding and Shou-Lai Gu contributed equally to this work.

Institute of Life Sciences, Jiangsu University, Zhenjiang, China
}

seed germination and seedling growth $[5,6]$. Seed oils mainly consist of triacylglycerols (TAGs), which comprise as much as $60 \%$ of the weight of a seed, and represent the most efficient form of energy storage in eukaryotic cells [7, 8]. Given the important role for TAGs, understanding the factors that limit their accumulation will contribute to increase TAG content by genetic engineering. In developing oilseeds, TAG biosynthesis takes place in the endoplasmic reticulum (ER). Carbohydrate and fatty acid (FA) metabolism are also involved in this pathway. In the cytosol and plastid, hexose is converted into acetyl-CoA through the glycolytic pathway, of which some are then used as the carbon source for FA synthesis. FAs can be converted to long-chain acyl-CoAs, which are precursors for TAG biosynthesis. Many genes that encode enzymes involved in glycolysis,

(c) The Author(s). 2020 Open Access This article is distributed under the terms of the Creative Commons Attribution 4.0 International License (http://creativecommons.org/licenses/by/4.0/), which permits unrestricted use, distribution, and 
as well as in FA and lipid biosynthesis play crucial roles in this pathway. Among them, the genes encoding longchain acyl-CoA synthetase (LACS, EC 6.2.1.3) catalyze the formation of acyl-CoA thioesters from free FAs in the presence of CoA, ATP, and $\mathrm{Mg}^{2+}$. It is a critical process in FA metabolism in prokaryotes and eukaryotes, and involves a two-step reaction. In the first step, free FAs react with ATP to generate a acyl-AMP, after which the acyl-CoA thioester bond is formed and AMP is released in the following step $[9,10]$. LACSs belong to the AMP binding protein (AMPBP) super family and mainly catalyze the synthesis of acyl-CoAs with acyl chain lengths of 12-20 carbons [11]. Previous studies have demonstrated that LACSs take an important position in nearly all FA-derived pathways, including lipid metabolism, jasmonate biosynthesis and $\beta$ oxidation $[12,13]$, as well as intracellular FA homeostasis and FA transport in various microorganism and mammalian cells [14-16]. In the diatom Phaeodactylum tricornutum, a lower photosynhetic organism, there were five putative LACSs (PtACSL1-5), and only two of which were able to restore growth, facilitate exogenous FA uptake, and enhance lipid accumulation in the yeast double mutant, FAA1 $\triangle F A A 4 \Delta$ [17]. Thus, those studies have provided a molecular basis for the study of LACSmediated FA and lipid metabolism in different organisms.

In higher plants, LACSs have several isoforms, which perform diverse biological functions in different cellular compartments [10, 18-21]. In Arabidopsis, there are nine $L A C S$ genes that participate in FA and glycerolipid metabolism. Seven of which could effectively complement the growth phenotype of a LACS-deficient yeast mutant strain, YB525. The two that could not complement the growth phenotype were peroxisomal isoforms [13]. AtLACS1 and AtLACS2 localize to the ER and have overlapping functions in wax and cutin synthesis with very long chain acyl-coenzyme A synthetase activity [22-24]. AtLACS1 and AtLACS4 showed a synergistic effect in the process of proper pollen coat formation [25]. AtLACS6 and AtLACS7 were involved in peroxisomal $\beta$ oxidation and successful seedling establishment $[10,26]$. LACS9 is localized to the chloroplast envelope and is involved in the production of acyl-CoA [21], and its function partially overlaps that of LACS1 and LACS4 in TAG biosynthesis and lipid trafficking from the ER to the plastid, respectively, in Arabidopsis [18, 27]. LACS homologs were also found in other plants, such as rapeseed, rice, soybean, and cotton, among others. A peroxisomal GmACSL2 from Glycine max is probably involved in FA and lipid degradation during seed germination [28]. Sunflower HaLACS1 and HaLACS2, which display sequence homology with the Arabidopsis LACS9 and LACS8 genes, respectively, were expressed at high levels in developing seeds and played an important role in sunflower oil synthesis [29].

Previous studies showed that there are six homologous $L A C S$ genes in $B$. napus, and two of which, including $B n L A C S 2$, have enzymatic activity when expressed in $E$. coli $[30,31]$. However, the biochemical properties and physiological functions of BnLACS2 remain uncharacterized. In this study, the B. napus BnLACS2 gene was cloned and its function in seed oil production was investigated. Overexpression of BnLACS2 in rapeseed plants resulted in improved oil content, while the suppression of gene expression in rapeseed decreased oil content. It was also shown that alterations in BnLACS2 function was associated with the physiological process of glycolysis, and FA and lipid synthesis in transgenic plants.

\section{Results}

\section{BnLACS2 is the orthologue of Arabidopsis LACS2 in} rapeseed

Using the protein sequence of AtLACS2 (NP_175368) as a query probe, two highly homologous sequences (BnaC05g51350D and BnaA05g16170D) were obtained from the Brassica genome database, and the putative full-length cDNA (2001 bp) of the BnaA05g16170D locus (designated as BnLACS2) was cloned from Zhongshuang 9 variety (Additional file 1: Figure S1). BnLACS2 contained an open reading frame consisting of 667 amino acid residues with a theoretical isoelectric point of 6.07 and a calculated molecular weight of $74.35 \mathrm{kDa}$. Sequence analysis revealed that BnLACS2 had a conserved AMP-binding domain, which was the characteristic domain of the AMPBP superfamily. Motif analysis of other LACS sequences using ScanProsite Results Viewer software also identified the notable AMP-binding domain, implying that this motif is functionally important for LACSs. The amino acid sequence of the BnLACS2 protein was then aligned with 24 LACS sequences from different species, including B. napus, Gossypium hirsutum, Arabidopsis thaliana, Saccharomyces cerevisiae, and Homo sapiens. A phylogenetic tree was constructed based on the amino acid sequences and revealed a close genetic relationship between BnLACS2 and Arabidopsis LACS2, suggesting that they might have similar functions (Fig. 1).

\section{BnLACS2 can complement the YB525 yeast mutant}

Previous research showed that $B n L A C S 2$ had in vitro enzymatic activity [31]; however, its exact function and mechanism of action have yet to be discovered. The yeast expression system is a powerful tool for the production of recombinant eukaryotic proteins. Moreover, yeast cells can accumulate oil, which is similar to rapeseed oil bodies [32]. Thus, the yeast expression system is suitable for investigating the function of genes in the 


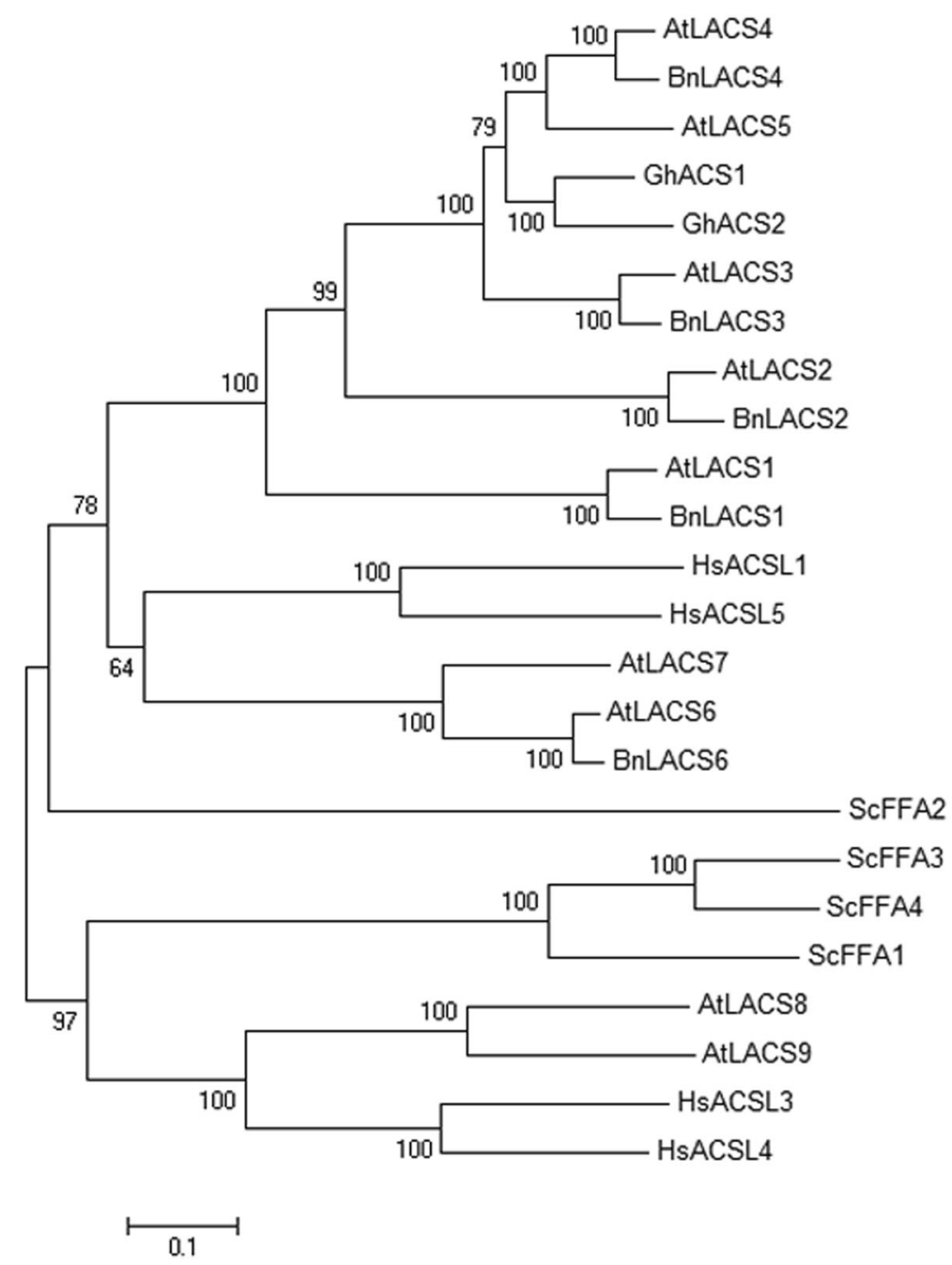

Fig. 1 Phylogenetic analysis of LACS protein family. The numbers beside the branches represent bootstrap on 1000 replications. The accession numbers are as follows: Arabidopsis thaliana AtLACS1 (NP_182246), AtLACS2 (NP_175368), AtLACS3 (NP_176622), AtLACS4 (NP_194116), AtLACS5 (AAM28872), AtLACS6 (NP_566265), AtLACS7 (NP_198112), AtLACS8 (NP_178516), AtLACS9 (NP_177882), Gossypium hisutum GhACS1 (ABA00144), GhACS2 (ABA00145), B. napus BnLACS1 (BnaC04g51420D), BnLACS2 (BnaA05g16170D), BnLACS3 (BnaC09g11030D), BnLACS4 (BnaC01g15670D), BnLACS6 (BnaA03g29320D), Homo sapiens HsACSL1 (NP_001986), HsACSL3 (NP_976251), HsACSL4 (NP_004449), HsACSL5 (NP_976313), Saccharomyces cerevisiae ScFFA1 (NP_014962), ScFFA2 (NP_010931), ScFFA3 (NP_012257), ScFFA4 (NP_013974). Bootstrapping was performed to obtain support values for each branch

lipid metabolism pathway. The YB525 yeast mutant lacks acyl-CoA synthetase activity, and provides a unique tool for the assessment of the functional roles of such enzymes [33]. Arabidopsis LACS2 exhibited enzymatic activity and could complement the LACS-deficient YB525 strain [13]. To determine whether BnLACS2 had a similar activity in vivo, BnLACS2 was cloned into pYES2 and transformed into YB525. The transformants were cultured in liquid medium with various FAs as the sole carbon source, including 12:0 lauric acid, 14:0 myristic acid, 16:0 palmitic acid, 18:0 stearic acid, 18:1 oleic acid, and 22:1 erucic acid. Then, the growth of those strains was evaluated based on optical density (OD). As shown in Fig. 2, transformation of pYES2-BnLACS2 rescued the growth defect of YB525 in medium containing 14:0, 16:0, 18:0, 18:1, and 22:1 FAs, but not 12:0 FAs, which is a short chain FA. However, yeast cells transformed with the pYES2 control vector failed to grow in cultures with FAs as the sole carbon source. Moreover, yeast cells containing the pYES2-BnLACS2 construct preferred to use 18:0 FAs as a carbon source for growth. Thus, BnLACS2 exhibited acyl-CoA synthetase activity and had a substrate preference for long chain FAs.

\section{Expression analysis of BnLACS2 in different tissues and different developmental stages of rapeseeds}

Although BnLACS2 could activate the free FA, its physiological function is unclear in rapeseed. Considering 


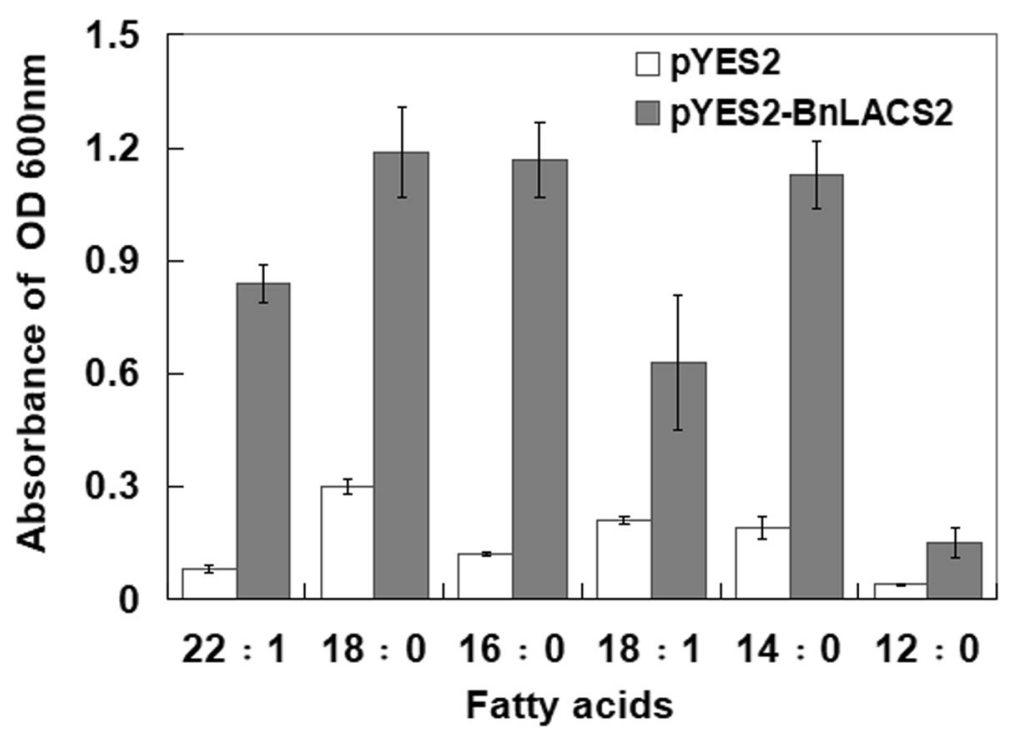

Fig. 2 The growth conditions of YB525 cells containing PYES2-BnLACS2 and pYES2 empty vector. The growth were measured for three lines of pYES2 and pYES2-BnLACS2 transformants cultured in the liquid medium used the fatty acid 12:0, 14:0, 16:0, 18:0, 18:1 and 22:1 as the sole carbon source, respectively. Error bars indicate SD $(n=3)$

that the tissue distribution of LACS genes may be closely related to their function, quantitative reverse transcription-polymerase chain reaction (qRT-PCR) was used to detect the expression patterns of the BnLACS2 gene in different rapeseed tissues, including roots, stems, leaves, flowers and developing seeds 25 days after pollination (DAP). The results showed that the BnLACS2 gene was expressed in all examined tissues, but was predominantly expressed in developing seeds where TAG was actively synthesized (Fig. 3a). Furthermore, there was an obvious difference in the abundance of BnLACS2 transcripts between high and low oil-content seeds at 25 DAP, 35 DAP, 45 DAP, and 50 DAP. Regardless of whether expression was evaluated in high or low oil-content seeds, BnLACS2 was strongly expressed at 45 DAP, which is the stage at which TAG is accumulated at a high rate (Fig. 3b). According to the transcription analysis results described above, BnLACS2 might be involved in supplying acyl-CoAs for TAG biosynthesis during seed development.

\section{Subcellular localization of BnLACS2}

In higher plants, LACS proteins with different subcellular localizations may participate in different metabolic pathways. It is known that the ER is an important site for FA carbon chain elongation and lipid synthesis. $A t \mathrm{LACS} 2$ is homologous to BnLACS2 and located in the ER. Therefore, we speculated that BnLACS2 is also an ER protein. To determine its localization, calreticulin 3 (CRT3) was selected as a marker for ER localization [34] and fused with a red fluorescent protein (DsRed) (Fig. 4a right). BnLACS2 was expressed as a fusion protein with green fluorescent protein (GFP) using the pK7FWG2.0 vector under the control of the CaMV $35 \mathrm{~S}$ promoter (Fig. 4a left). The plasmids, 35S::BnLACS2-GFP and 35S:: DsRed-CRT3, were generated and transfected into the leaf epidermal cells of Nicotiana benthamiana for transient expression. As shown in Fig. 4b-d, the GFP fluorescence produced by the BnLACS2-GFP fusion protein was found to overlap with the DsRed fluorescence produced by DsRed-CRT3 fusion protein, indicating that $B n L A C S 2$ was co-localized with CRT3 in the ER, i.e., the site of TAG biosynthesis.

\section{BnLACS2 expression is correlated with lipid and FA contents in transgenic yeast and rapeseed}

Expression pattern and subcellular localization analyses of $B n L A C S 2$ suggested that it may be involved in seed oil production. The function of BnLACS2 was first identified with S. cerevisiae pep4 mutants with at least $90 \%$ reduced activity of the four vacuolar proteinases [35]. The Sudan black B staining method was used to indicate the relative content of neutral lipids in host yeast cells. The results showed that the absorbance values of BnLACS2-transformed lines increased by $85.7 \%$, compared to the control cell transformed with empty pYES2. Moreover, BnLACS2 overexpression resulted in producing new phospholipid components in yeast. According to gas chromatography-mass spectrometry (GC-MS) analyses, heterologous expression of BnLACS2 also significantly enhanced the synthesis of total FAs, and in particular, two main FA components, palmitoleic acid (C16:1) and oleic acid (C18:1) (Additional file 2: Figure S2). 


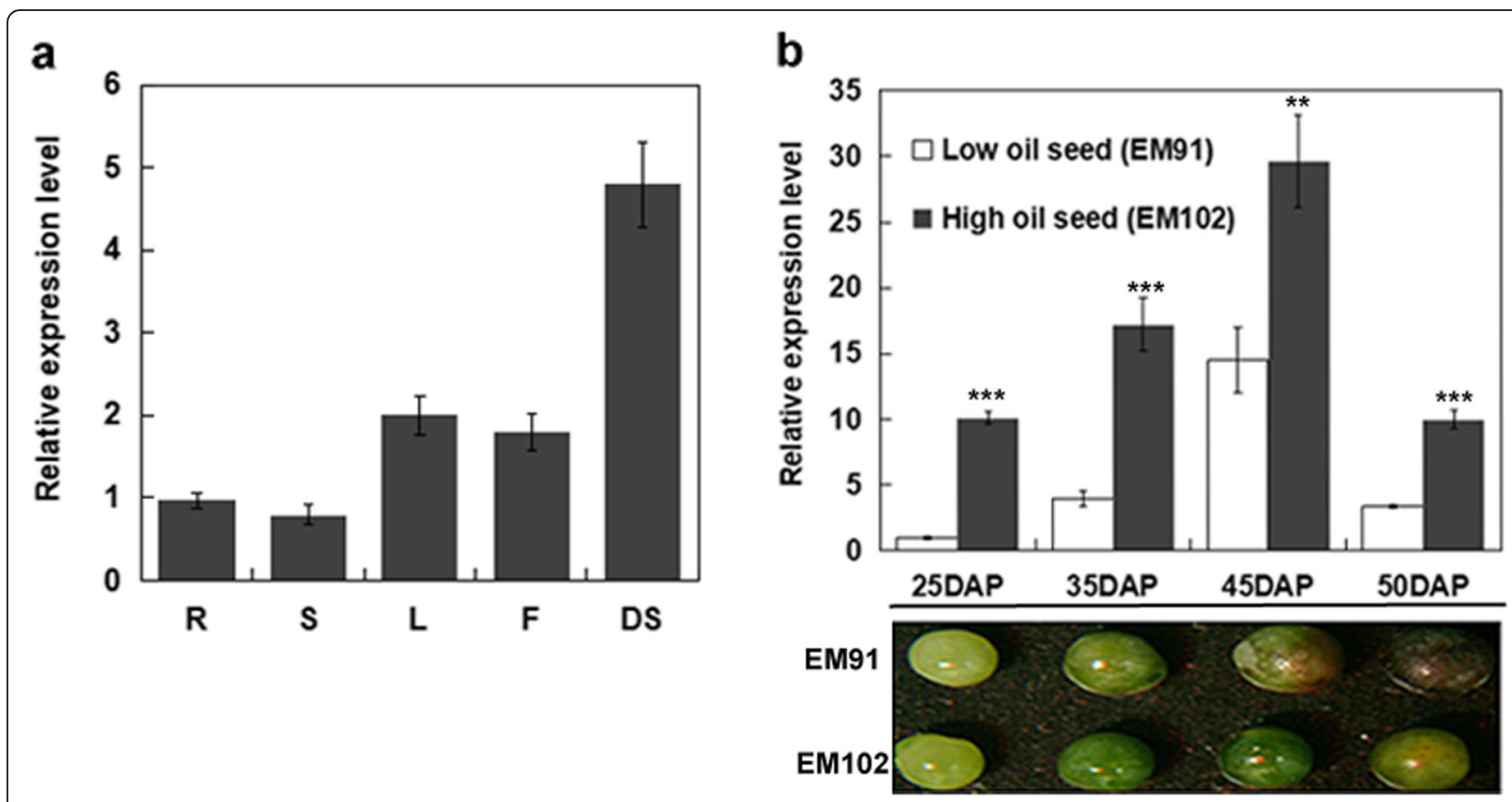

Fig. 3 Expression patterns of BnLACS2. a qRT-PCR analysis of BnLACS2 in different tissues, including root (R), stem (St), leaf (L), flower (F), developing seed (DS, DAP25). The expression levels were quantified relative to the value obtained from root. $\mathbf{b}$ Expression patterns of BnLACS2 in low (EM91) and high (EM102) oil-content seeds of different developmental stages. The photographs were taken during the developing of seeds at 25 DAP, 35 DAP, 45 DAP, and 50 DAP. The expression levels were quantified relative to the value obtained from EM91 (25 DAP). Error bars represent SD $(n=3)$. The significant differences between EM91 and EM102 at each time point (25 DAP, 35 DAP, 45 DAP, and 50 DAP) are indicated (Student's $t$-test) as follows: ${ }^{* *}, P<0.001 ;{ }^{* *}, P<0.01 ;{ }^{*}, P<0.05$

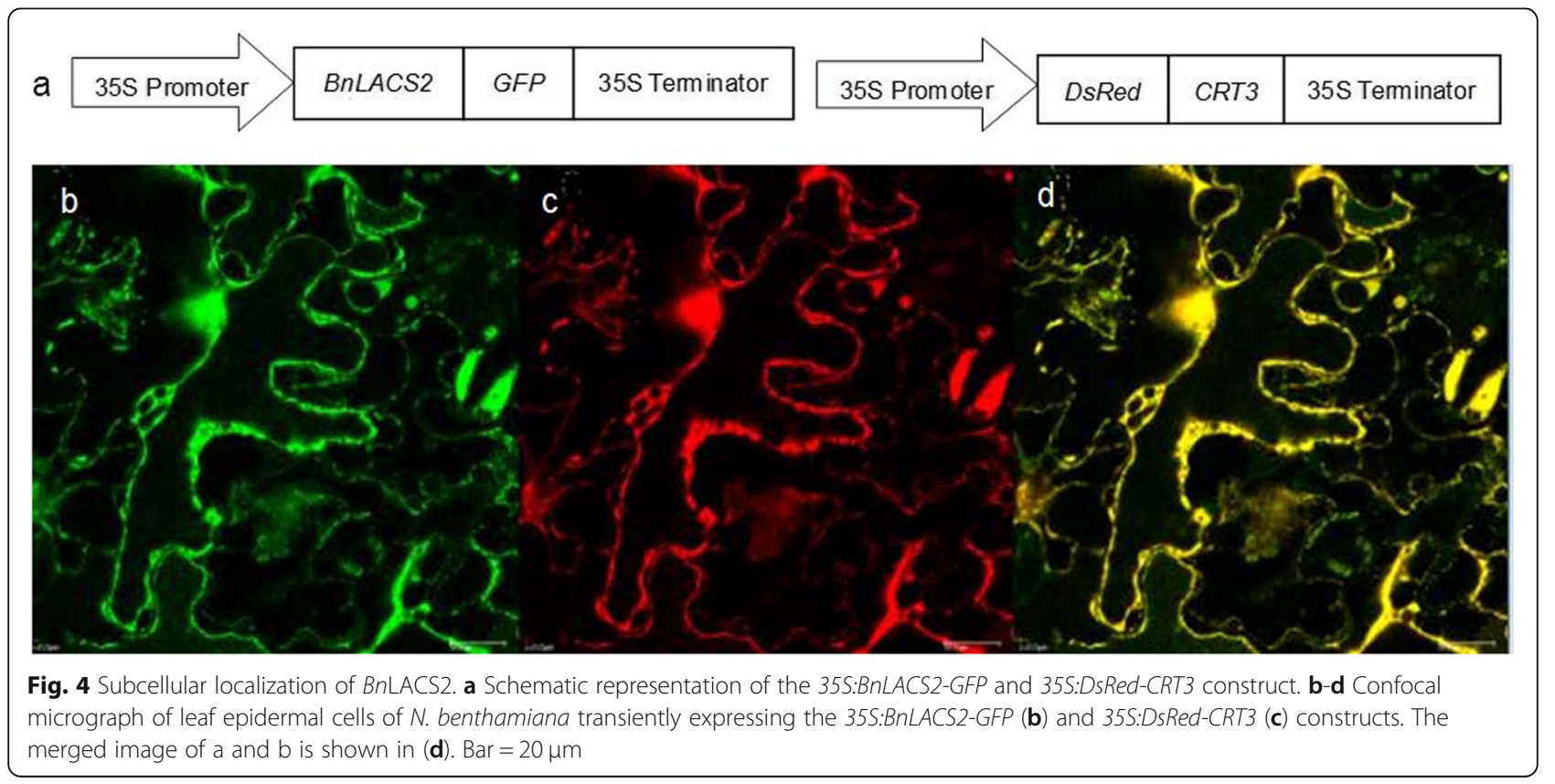


To further confirm the function of $B n L A C S 2$, vectors for BnLACS2-overexpression (OE) and BnLACS2-RNAi were constructed and transformed into rapeseed $(B$. napus L. cv. Zhongshuang 9) using the floral dip method (Fig. 5a). The preliminary screening of hygromycin- or kanamycin-resistant rapeseed transformants was performed via PCR. Several independently transformed T0 transgenic plants were selected and grown to maturity to obtain homozygous lines (Fig. 5b). The detection of the BnLACS2 transcripts by qRT-PCR in T2 transgenic lines revealed that its expression level was higher in $B n L A C S 2-O E$ transgenic plants than in untransformed control plants, while expression was lower in BnLACS2RNAi transgenic plants, compared to the controls (Fig. $5 \mathrm{c})$. The phenotypes of BnLACS2-OE and BnLACS2RNAi transgenic plants were also analyzed. Compared to non-transgenic plants, transgenic plants had normal phenotypes and exhibited no obvious changes in morphology. To further determine whether BnLACS2 was involved in seed TAG biosynthesis, we analyzed the seed oil content in $\mathrm{T} 2$ transgenic rapeseed plants using near infrared spectrometry (NIRS) combined with nuclear magnetic resonance (NMR), and obtained consistent results between these two methods. As shown in Tables 1 and 2, the oil content in BnLACS2-OE plants was increased by 6 to $8 \%$, and decreased by 3 to $6 \%$ in BnLACS2-RNAi transgenic plants, compared to wild type plants. Moreover, GC-MS analysis showed that overexpression of BnLACS2 also significantly enhanced the contents of long-chain FAs, such as C18:2, C20:0,
C20:1 and C22:0 (Fig. 6). These findings suggested that the expression of BnLACS2 in rapeseed and yeast cells increased the content of lipids and FAs. Thus, as an important enzyme for the catalysis of free FAs to longchain acyl-CoAs, BnLACS2 may play an essential role in seed oil production in rapeseed.

\section{BnLACS2 overexpression influences the expression of genes involved in glycolysis in developing seeds}

The expression of BnLACS2 in yeast pep 4 could increase lipid and FA content. To further characterize the effect of BnLACS2 in the control of lipid and FA synthesis, the proteins of pYES2 and pYES2-BnLACS2 transformants were separated using two-dimensional gel electrophoresis (2-DE). The results revealed that some glycolytic enzymes were upregulated in pYES2-BnLACS2 transformants (Additional files 3 and 4: Figure S3 and Table S1). The upregulation of glycolytic enzymes caused by the expression of BnLACS2 in yeast prompted us to then detect whether these genes were altered in BnLACS2 transgenic rapeseed plants. qRT-PCR analysis revealed that the expression of genes for key glycolytic enzymes, including phosphofructokinase (PFK), phosphoglycerate kinase (PGK), enolase (ENO) and pyruvate kinase (PYK) [36-38] were significantly increased in BnLACS2-OE transgenic plants, while the expression of those genes were decreased in BnLACS2-RNAi transgenic plants, compared to non-transgenic plants (Fig. 7). This result indicated that the levels of BnLACS2 affect the expression of glycolytic genes in developing seeds.

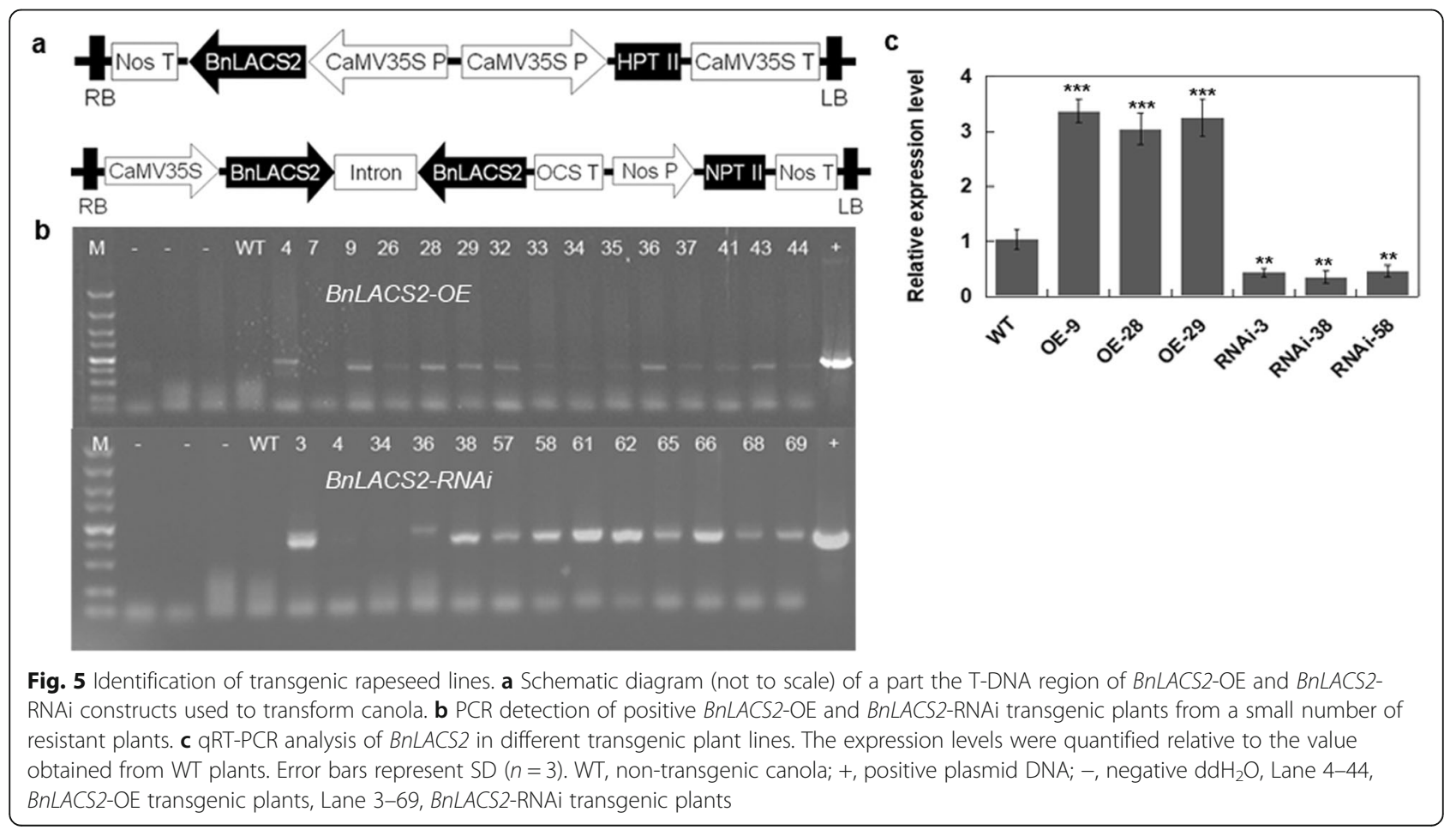


Table 1 Canola oil content is determined in NMR

\begin{tabular}{llll}
\hline Line & Lipid content 1(\%) & Lipid content 2(\%) & Average of lipid content (\%) \\
\hline WT & 38.99 & 38.94 & 38.97 \\
BnLACS2-OE9 & $42.28^{*}$ & $42.01^{*}$ & $42.15^{*}$ \\
BnLACS2-OE28 & 41.14 & 41.33 & 41.37 \\
BnLACS2-OE29 & $42.04^{*}$ & $41.98^{*}$ & $42.01^{*}$ \\
BnLACS2-RNAi3 & $36.98^{*}$ & $36.84^{*}$ & $36.91^{*}$ \\
BnLACS2-RNAi38 & 37.83 & 37.77 & 37.80 \\
BnLACS2-RNAi58 & $36.87^{*}$ & $36.60^{*}$ & $36.74^{*}$ \\
\hline
\end{tabular}

The values are the mean percentages of each genotype $(n=3)$. "Statistically significant difference from WT (Students't test, $P<0.05)$

High levels of BnLACS2 activity modifies the expression of FA and lipid synthesis genes in developing seeds

From the above results, we found that modification of BnLACS2 activity levels affected glycolytic gene expression, but the mechanism for that effect is unknown. To further understand the role of BnLACS2 in the control of seed oil production, the expression level of several representative genes in the FA and lipid biosynthesis pathways were also examined in developing seeds, including acetyl-CoA carboxylase 1 (ACC1), beta-ketoacyl[acyl carrier protein] (ACP) synthase (KAS II), FATTY ACID ELONGATION1 (FAE1), FATTY ACID DESATURASE 3 (FAD3), lysophosphatidyl acyltransferase 1 (LPAT1), and diacylglycerol acyltransferase 1 (DGAT1). Among those genes, ACC1 and KASII are involved in the FA condensation reaction $[39,40]$, and $F A E 1$ and $F A D 3$ participate in FA chain elongation and desaturation reactions, respectively [41, 42]. LPAT1 and DGAT1 are related to lipid biosynthesis $[43,44]$. Expression profile analysis showed that those genes were all significantly increased in BnLACS2-OE transgenic plants and decreased in BnLACS2-RNAi transgenic plants, compared to wild type plants (Fig. 8). These results suggested that high levels of BnLACS2 could modify the expression of FA and lipid synthesis genes in developing seeds and play an important role in seed oil production, although other LACS activities should be involved due to the limited observed changes $( \pm 10 \%$ of the total oil content).

\section{Discussion}

Oilseed rape is a major oil crop in the world and plays important roles in economy and agricultural production. Increasing seed oil content is of great economic value and a challenge for rapeseed breeding programs. LACSs are important factors for FA and lipid metabolism. Many LACS proteins have been studied in the model plants, Arabidopsis and rice [13, 45]. However, functional characterizations of LACS proteins in rapeseed have been rarely reported. In the present study, the LACS gene, $B n L A C S 2$ was identified and characterized from rapeseed (Zhongshuang 9). We showed that BnLACS2 encodes a LACS protein, which can restore normal growth in a LACS-deficient yeast strain (YB525) upon complementation. Moreover, overexpression of BnLACS2 in yeast and oilseed rape could significantly increase lipid content. Thus, BnLACS2 is a candidate gene for highoil-content breeding in rapeseed.

$B n L A C S 2$ contained the conserved AMP-binding domain that is typical of the AMPBP gene superfamily, which share a mechanism step in carboxylic acid activation via adenylation [46]. Phylogenetic analyses showed that BnLACS2 was homologous to Arabidopsis AtLACS2 (Fig. 1), which has been shown to rescue growth defects of LACS-deficient yeast (YB525) [13]. Based on the sequence homology between BnLACS2 and AtLACS2, it was speculated that they may exhibit similar functions. As a result, it was shown that heterogeneous expression of BnLACS2 could also complement the YB525 yeast

Table 2 Canola oil content is determined by NIRS

\begin{tabular}{|c|c|c|c|c|c|c|c|c|c|}
\hline Line & Oleic acid & Linoleic acid & Linolenic acid & Erucic acid & SFA & Thioglycoside & Protein & Water & Lipid \\
\hline$\overline{W T}$ & 59.98 & 21.47 & 6.12 & 0.21 & 6.59 & 15.59 & 25.49 & 7.34 & 37.62 \\
\hline BnLACS2-OE9 & 61.10 & 20.55 & 7.22 & 0.11 & 6.76 & 16.29 & $21.98^{*}$ & 7.37 & 39.64 \\
\hline BnLACS2-OE28 & $57.10^{*}$ & $19.39^{*}$ & 6.32 & $0.37^{*}$ & 6.56 & $11.68^{*}$ & $23.31^{*}$ & 6.95 & $40.05^{\circ}$ \\
\hline BnLACS2-OE29 & 60.96 & 19.81 & 6.62 & $0.44^{*}$ & 6.71 & $13.16^{*}$ & $21.42^{*}$ & 7.13 & $40.40^{\circ}$ \\
\hline BnLACS2-RNAi3 & $56.26^{*}$ & $23.32^{*}$ & 6.79 & 0.28 & 6.78 & 17.12 & 24.84 & 7.69 & 35.34 \\
\hline BnLACS2-RNAi38 & $56.41^{*}$ & 21.54 & 7.79 & $0.53^{*}$ & 6.45 & $23.89^{*}$ & 26.24 & 7.35 & 36.69 \\
\hline BnLACS2-RNAi58 & $57.21^{*}$ & $23.16^{*}$ & 6.88 & $0.46^{*}$ & 6.67 & $18.88^{*}$ & 24.90 & 7.85 & 35.35 \\
\hline
\end{tabular}

The values are the mean counts of triplicate determinations. "Statistically significant difference from WT (Students't test, $P<0.05$ ) 


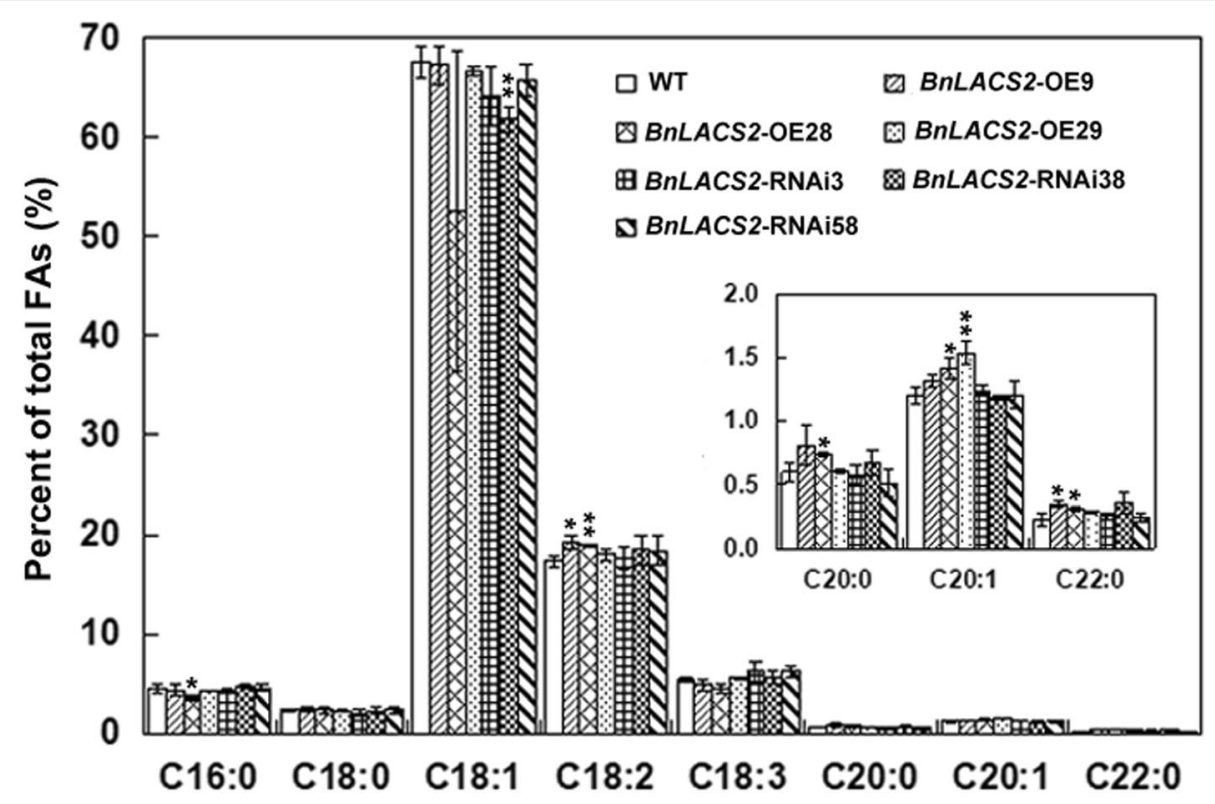

Fig. 6 Analysis of FA contents in WT, BnLACS2-OE and BnLACS2-RNAi transgenic seeds. Mature $T_{2}$ generation seeds were used for the analysis. Error bars represent SD $(n=3)$. The significant differences between transgenic lines and WT are indicated (Student's $t$-test): ${ }^{* *}, P<0.01 ;{ }^{*}, P<0.05$
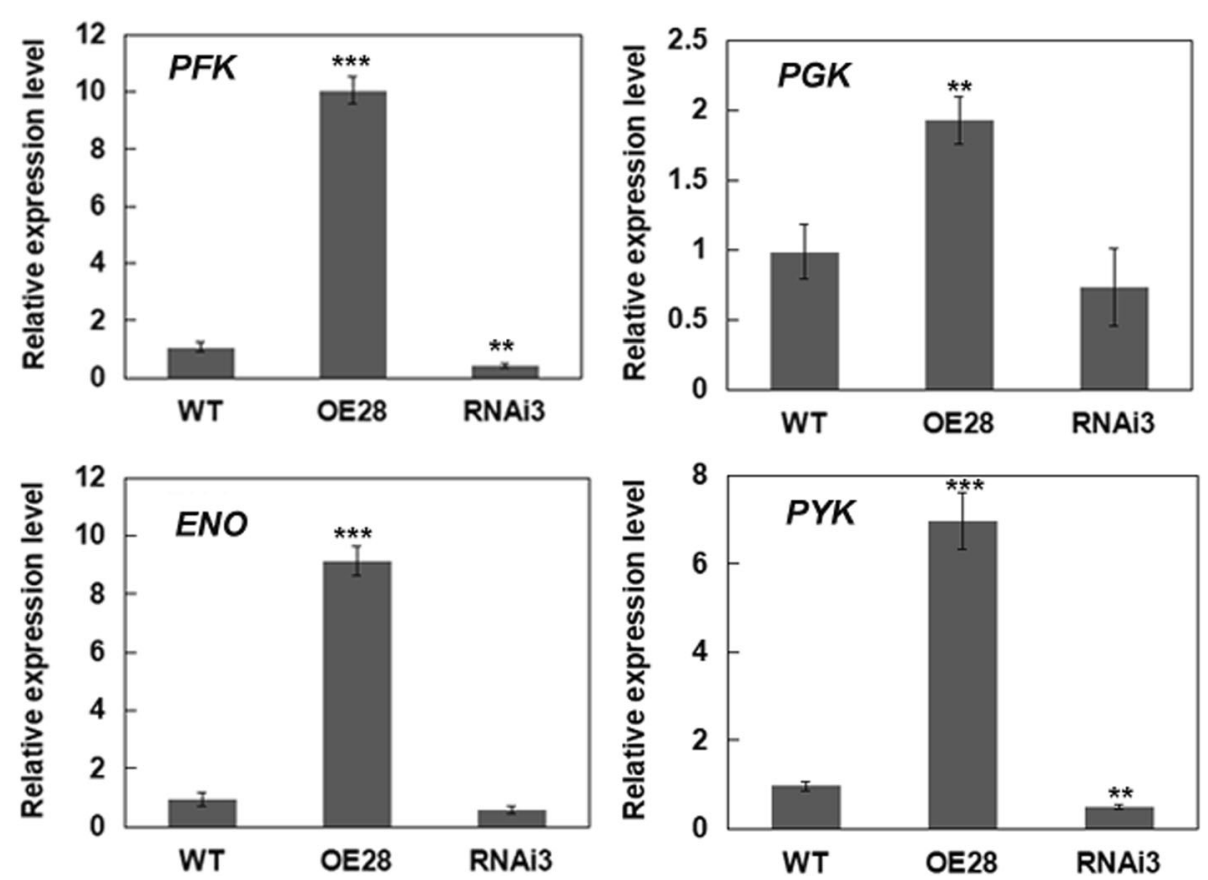

Fig. 7 Expression of representative glycolytic genes in developing seeds of the transgenic canola plants. qRT-PCR was performed using total RNA prepared from developing seeds (40 DAP) collected from the WT and transgenic plants. The expression levels were quantified relative to the value obtained from WT plants. Error bars represent SD $(n=3)$. The significant differences between transgenic lines and WT are indicated (Student's $t$-test): ${ }^{* * *}, P<0.001 ;{ }^{* *}, P<0.01 ;{ }^{*}, P<0.05$ 

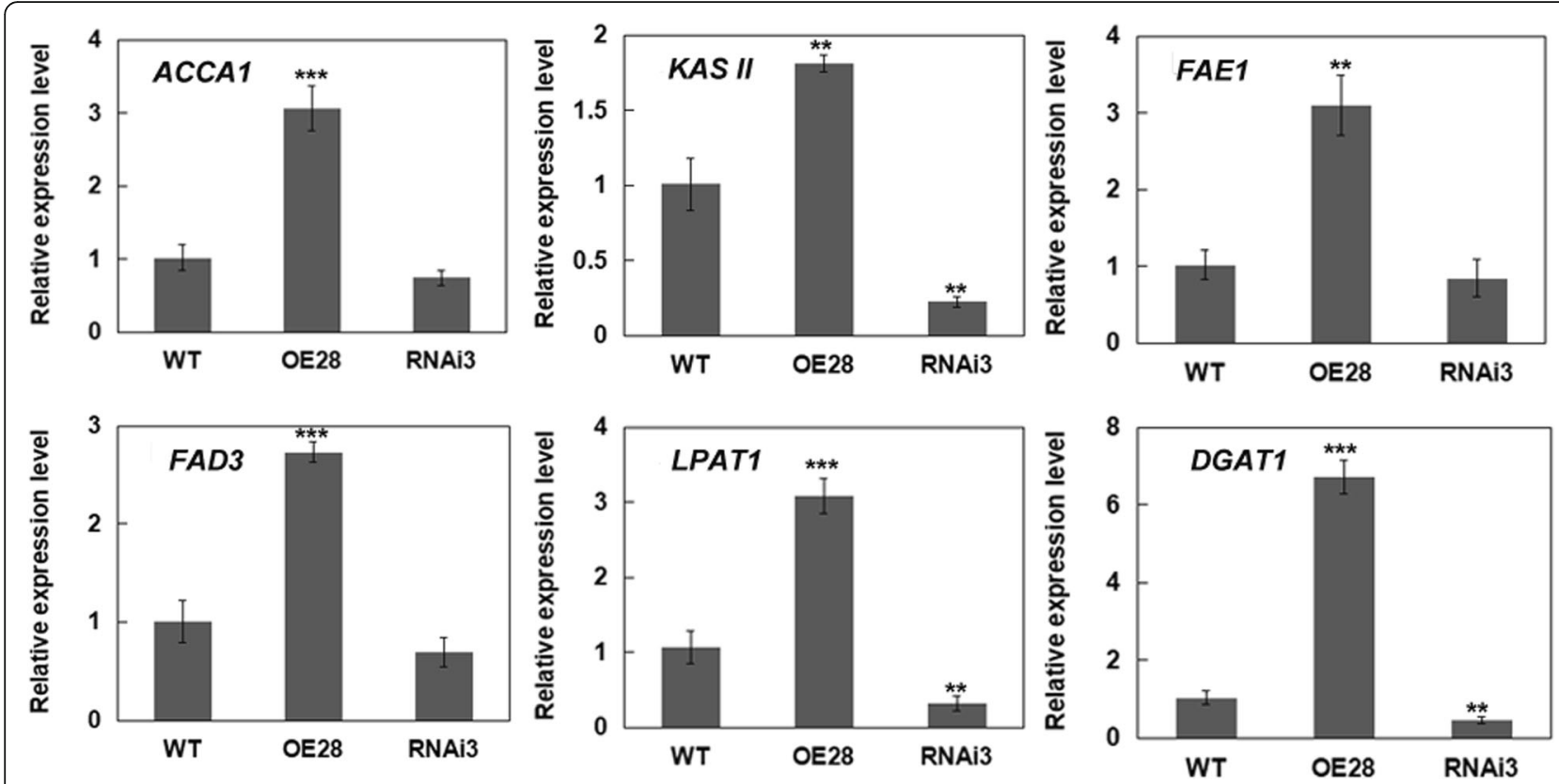

Fig. 8 Expression of representative FA and lipid synthetic genes in developing seeds of the transgenic canola plants. The expression levels were quantified relative to the value obtained from WT plants. Error bars represent SD $(n=3)$. The significant differences between transgenic lines and WT are indicated (Student's t-test): ${ }^{* *}, P<0.001 ;{ }^{*}, P<0.01 ; *, P<0.05$

mutant. The yeast complementation test not only confirmed the long-chain acyl-CoA synthetase activity of $B n L A C S 2$, but also revealed its substrate preference and specificity. When YB525 yeast cells were transformed with BnLACS2, they were able to survive in auxotrophic medium containing FAs of different carbon chain lengths $(\mathrm{C} 12-\mathrm{C} 22)$. The data revealed that the growth defect associated with the YB525 was rescued with long chain FAs (C14-C22) as the carbon source when $B n L A C S 2$ was expressed, but not with short chain FAs (C12). These results indicated that BnLACS2 exhibited high acyl-CoA synthetase activity and played an important role in the activation of FAs to acyl-CoA. Furthermore, BnLACS2 preferably utilized long chain FAs as the substrates (Fig. 2), and therefore, BnLACS2 is a long chain acyl-CoA synthetase gene.

Multiple studies reported that several plant LACSs exhibited different expression patterns in different tissues $[13,20,28,29]$. In our study, BnLACS2 was predominantly expressed in developing seeds where TAG was actively synthesized (Fig. 3a). Further analyses of BnLACS2 expression patterns from 25 DAP to 50 DAP of embryo development showed that BnLACS2 was strongly expressed at 45 DAP, which is the point at which TAG accumulates at a high rate (Fig. $3 \mathrm{~b}$ ). Those data suggested that BnLACS2 may be involved in metabolic processes related to seed oil production. It has been reported that LACS proteins in plants may be localized to different organelle fractions to determine the function of LACS proteins [18, 30, 47]. Consistent with the above data, subcellular localization analyses showed that GFP-tagged BnLACS2 and the DsRed-tagged ER localization marker $C R T 3$ were co-localized, indicating that BnLACS2 was localized to the site of TAG biosynthesis (Fig. 4). Similarly, a few plant LACS proteins localized in the ER were reported to play roles in TAG biosynthesis, including Arabidopsis LACS1 and sunflower LACS2 [27, 29]. Therefore, BnLACS2 may participate in the activation of long chain FAs for lipid synthesis during seed development. Moreover, BnLACS2 was expressed at higher levels in leaves, which was consistent with that of Arabidopsis LACS2 in cutin biosynthesis [23, 24]. However, the function of AtLACS2 in seed oil biosynthesis remains unclear.

To assess the contribution of BnLACS2 in TAG production, BnLACS2-OE and BnLACS2-RNAi transgenic plants were generated and their seed oil contents were analyzed. The results indicated that transgenic rapeseed plants overexpressing the BnLACS2 gene exhibited higher oil content in seeds, compared to WT, while RNAi-mediated BnLACS2 silencing resulted in lower oil contents, compared to WT plants (Tables 1 and 2). Similarly, the expression of BnLACS2 in pep4 yeast significantly increased lipid and FA contents (Additional file 2: Figure S2). Thus, these results revealed that $B n L A C S 2$ was involved in TAG biosynthesis. Moreover, both BnLACS2-OE and BnLACS2-RNAi transgenic 
plants had normal growth and development. Therefore, BnLACS2 may be a good candidate gene for increasing the seed oil content of rapeseed, without imparting negative effects.

The 2-DE analysis of yeast proteins showed that heterologous expression of $B n L A C S 2$ resulted in expression profile alterations associated with glycolysis (Additional file 4: Table S1). In transgenic rapeseed plants, the overexpression of BnLACS2 also affected the expression of glycolytic genes (Fig. 7). Moreover, the increased glycolytic activity was accompanied by increases in lipid content in yeast and BnLACS2 transgenic plants, which may be due to the increased carbon flux to the FA biosynthesis pathway. In the cytosol and plastid, hexose is converted to acetyl-CoA through the glycolytic pathway, and some of which is then used as the carbon source for FA synthesis. This result implied that BnLACS2 could also affect FA and lipid biosynthesis. Therefore, it is unsurprising that as a consequence of $B n L A C S 2$ regulation, genes involved in FA and lipid synthesis pathways, including ACC1, KASII, FAE1, FAD3, $L P A T 1$, and DGAT1, were upregulated in BnLACS2 transgenic plants, and downregulated in BnLACS2-silenced plants (Fig. 8).

Previous research demonstrated that the transport of FAs involves two processes, including de novo synthesis of plastidial FAs in the form of acyl-ACP, followed by releasing from ACP by a thioesterase, and subsequent export from plastids in the form of acyl-CoA by LACS action; thus, providing an acyl-donor for TAG assembly in the ER [48-50]. Therefore, LACSs mediate de novo FA synthesis in the plastids and lipid biosynthesis in the ER $[29,48]$. In addition to the important role of acylCoA esters in TAG synthesis, they also serve as metabolic intermediates in acyl chain elongation reactions, $\beta$ oxidation, and other lipid metabolism in plants [45]. It is generally considered that the acyl-CoAs that are synthesized on the outer mitochondrial and peroxisomal membranes are used for oxidation [28, 51], while those synthesized in the ER play synthetic roles [27]. However, the partitioning of acyl-CoAs within cells remains unclear. In this study, BnLACS2 was targeted to the ER, suggesting that it was not required for the transport of FAs through the plastid. Meanwhile, the lipid and FA contents of the pep4 yeast mutant were improved upon BnLACS2 overexpression; thus, suggesting that BnLACS2 had the capacity to supply acyl-CoA substrates for TAG synthesis [52]. To balance acyl-CoA pools in the cytoplasm and ER, it is speculated that some acyl-CoA esters are exported to the ER while releasing the CoA in the cytoplasm. Then, long-chain FAs are reactivated to form acyl-CoA esters by LACS, such as BnLACS2; thus, contributing to TAG accumulation in the ER. However, it is just a possible functioning manner by which the overexpression of BnLACS2 causes increases in lipid contents in yeast and rapeseed, and additional studies are required to confirm this deduction.

\section{Conclusions}

In the present study, BnLACS2, an orthologue of the Arabidopsis LACS2 gene, was characterized as an important factor determining the seed oil content in the tetraploid, B. napus. BnLACS2 overexpression increased oil content in rapeseed, while its silencing by RNAi decreased oil contents, compared to wild type plants. Furthermore, the altered levels of BnLACS2 were correlated with an enhancement or attenuation of glycolysis, and the FA and lipid synthesis pathways in transgenic plants. These results suggested that the BnLACS2 gene is a potential target for increasing seed oil production in rapeseed via genetic manipulation efforts. Based on our data, we speculate that BnLACS2 levels modify three metabolic pathways that function in different parts of the cell, including glycolysis, FA synthesis, and lipid synthesis. However, the precise molecular mechanism by which $B n L A C S 2$ influences the expression of genes involved in those physiological processes should be further studied.

\section{Methods}

Plants materials and growth condition

Plants of B. napus (Zhongshuang 9) were obtained from the Oil Crops Research Institute (OCRI) of the Chinese Academy of Agricultural Sciences (CAAS) and were grown in the experimental fields of Jiangsu University, Zhenjiang, China. For tissue-specific expression analyses, rapeseed roots, stems, leaves, flowers and developing seeds were collected and stored at $-70^{\circ} \mathrm{C}$. Low (EM91) and high (EM102) oil-content canola lines were obtained from State Key Laboratory of Crop Genetics and Germplasm Enhancement, Nanjing Agricultural University, China. The seed tissues were harvested at 25, 35, 45, and 50 DAP. Nicotiana benthamiana stored in our laboratory were grown in a plant growth room for 5-6 weeks under a temperature-adjusted daytime/night cycle of 22/ $20^{\circ} \mathrm{C}$, and an illumination time of $16 \mathrm{~h} / \mathrm{d}$.

\section{Sequence analysis of BnLACS2}

The full length coding region of BnLACS2 was obtained using the protein sequence of AtLACS2 (NP_175368) to search Brassica genome sequence databases (http:// www.genoscope.cns.fr/blat-server/cgi-bin/colza/webBlat). Protein properties, including molecular weight, isoelectric point, sequence length, and amino acid composition were determined using the ProtParam tools in the ExPASy database (http://web.expasy.org/protparam/). Domain analysis was performed using the CDD database in the National Center for Biotechnology Information (NCBI, http://www. ncbi.nlm.nih.gov) and the ScanProsite Results Viewer in ExPASy (http://us.expasy.org/prosite/). The amino acid 
sequences of plant LACS were retrieved from nonredundant protein sequence databases using the blast method in NCBI and Brassica genome sequence databases. Multiple alignment of the amino acid sequences of LACS homologs were analyzed using the ClustalW program [53]. Phylogenetic analyses were performed using the Neighbor-joining algorithm in MEGA 5.0 software [54].

\section{Subcellular localization of BnLACS2}

Total RNA was extracted from the developing seeds of Zhongshuang 9 using the Trizol RNA Preparation Kit (Invitrogen, USA), following the manufacturer's protocol. First-strand cDNA was synthesized using $1-2 \mu \mathrm{g}$ of total RNA as the template, oligo (dT) as the primers, and reverse transcriptase (Fermentas). The cDNA of the $B n L A C S 2$ gene was amplified by PCR using the primers, 5' -ATGTACACAAGCGGGACGAC-3' and 5' -ACGCCA GTATCCAACAGAGG-3', and cloned into the pMD18 T plasmid. To observe the subcellular localization of $B n L A C S 2$, translational fusions of BnLACS2-pK7FWG2.0 and CRT3-pCX-DR were constructed and transformed into Agrobacterium tumefaciens strain GV3101 using the freeze-thaw method described in Karimi et al. [55]. The subcellular distribution of the transiently expressed $35 S$ : BnLACS2-GFP and 35S:DsRed-CRT3 in N. benthamiana was observed using a Leica TCS scanning confocal microscope, as described previously $[56,57]$.

\section{Yeast transformation and complementation}

The cDNA of the BnLACS2 gene was amplified using the primers, F: 5'-ggtaccATGGCTGCAGCTGCTGATCATG$3^{\prime}$ and R: 5'-ggatccCTAGCTCTGGACGCCTTTGCTTC$3^{\prime}$, and inserted into the multiple cloning sites (Kpn I/ $B a m H$ I) of pYES2 to generate the pYES2-BnLACS2 vector. The recombinant plasmid, pYES2-BnLACS2, and the pYES2 empty vector were introduced into the YB525 yeast strain by uracil auxotrophy $[13,58]$. Then, positive recombinant clones were screened on Synthetic Dropout Medium-Uracil (SC/ura ${ }^{-}$) plates supplemented with $0.67 \%$ $(\mathrm{w} / \mathrm{v})$ Yeast nitrogen base lacking amino acids, with $0.077 \%$ $(w / v)$ uracil default mixture, and $2 \%(w / v)$ glucose. Single colonies were randomly chosen and cultured in SC/ura- liquid medium to the logarithmic growth phase. Cells were then collected and incubated in 3-5 $\mathrm{mL}$ SC/ura ${ }^{-}$liquid medium with $2 \%$ galactose for $4 \mathrm{~h}$. After adjusting the cell concentration to a uniformity, $30 \mu \mathrm{L}$ cultures were pipetted onto $3 \mathrm{ml} \mathrm{SC} /$ ura $^{-}$liquid medium containing $2 \%$ galactose plus $98 \mu \mathrm{M}$ of different FAs, including 12:0 lauric acid, 14:0 myristic acid, 16:0 palmitic acid, 18:0 stearic acid, 18:1 oleic acid and 22:1 erucic acid dissolved in $0.1 \%$ Triton X-100 as the sole carbon source. Cultures were then cultivated for $84 \mathrm{~h}$ at $30^{\circ} \mathrm{C}$. The growth of yeast cells was estimated based on absorbance at $600 \mathrm{~nm}$ using a spectrophotometer.

\section{Analysis of lipids and FAs in yeast pep4}

The recombinant vector, pYES2-BnLACS2, and the pYES2 control were transformed into pep4, a proteinasedeficient mutant strain. Screening of positive yeast monoclonal and induction of the BnLACS2 protein are consistent with the methods described above. Yeast cell concentrations were adjusted to the same concentration based on the optical density at $600 \mathrm{~nm}$. As described by Thakur et al. [59], equivalent volumes of yeast suspensions were then centrifuged to collect the cells, and stained with $0.3 \%$ Sudan black B to detect the neutral lipid content. The lipids were extracted from yeast cells using chloroform and methanol method as described by Bligh and Dyer [60]. Two dimensional thin layer chromatography (2D-TLC) was performed on $20 \times 20 \mathrm{~cm}$ silica gel 60F254 aluminum sheets (Merck, Germany). The solvents were chloroform/methanol/water (65:30:2.5, v/ v) in the first dimension and chloroform/methanol/ acetic acid/water (80:12:15:4, v/v) in the second dimension. The polar lipids were detected by staining with chromogenic reagents as described by Guan et al. [61].

FAs were methylated following the procedure of Brandenburg et al. [62]. Prior to GC-MS analysis, $10 \mu \mathrm{l}$ methylheptadecanoate $(10 \mu \mathrm{g} / \mu \mathrm{l}$, Sigma-Aldrich, USA) was added into the extracted lipid samples as an internal standard. FA profiles were determined using GC-MS equipped with a $30 \mathrm{~m} \times 0.25 \mathrm{~mm}$ BPX 70 fused-silica capillary column (SGE, Austin, TX, USA), as previously described [63]. After the identification of each FA species based on their specific retention times, the FAs were quantified using the internal standard.

\section{Proteomics analysis of the pep4 yeast strain transformed with the BnLACS2 gene}

After induction of the pYES2-BnLACS2 and pYES2 vectors, total proteins were extracted from the pep4 yeast strain using a Tris buffer-based protocol, as described by Isola et al. [64]. Protein concentration was determined by the Bradford method [65]. 2-DE was performed as described in Ding et al. [66], with a few minor changes. Protein samples were isolated in $17 \mathrm{~cm} \mathrm{pH} \mathrm{3-10} \mathrm{linear}$ gel strip (Bio-Rad). Isoelectric focusing procedure was set at 250,500 and $2000 \mathrm{~V}$ for $1 \mathrm{~h}$, respectively, and then $8000 \mathrm{~V}$ for $56,000 \mathrm{Vh}$. After equilibration and the second dimension sodium dodecyl sulfate polyacrylamide gel electrophoresis was performed, gels were visualized using Coomassie Brilliant Blue R-250 (0.1\%) staining and scanned using a SanMaker 9700XL instrument (BioRad). Image analysis using PDQuest software (Bio-Rad) and in-gel digestion were performed as described in Ding et al. [67].

Spots with statistically significant changes (Student's $t$ test, $P<0.05)$ above a 2 -fold threshold were selected for 
mass spectrometric (MS) analysis using the UltrafleXtreme MALDI TOF/TOF instrument (Bruker Daltonics, Billerica, MA, USA). Mass data were acquired in the positive ion reflector mode. To identify differentially expressed proteins, the peptide mass fingerprint was searched in the NCBI non redundant (nr) database using Mascot software (http://www.matrixscience.com). The following search parameters were used: taxonomy, fungi; trypsin; one missed cleavage allowed; fixed modification, carbamidoethyl; variable modifications, dioxidation; peptide tolerance, $\pm 0.2 \mathrm{Da}$; and peptide charge, $1+$. Protein scores $>65$ were considered significant $(P<0.05)$.

\section{Generation of transgenic rapeseed plants}

To generate CaMV 35S-driven constructs, the fulllength coding region of BnLACS2 was amplified using primers 5' -ggtaccATGGCTGCACTGCTGATCATG-3' and $5^{\prime}$-ggatccCTAGCTCTGGACGCCTTTGCTTC-3'. The resulting fragment was digested with $\mathrm{BamH} \mathrm{I}$ and $K p n I$, and then inserted into the same sites of the pCAMBIA1300-35S-Nos vector. To construct the $B n L A C S 2-R N A i$ plasmid, partial fragments of BnLACS2 were amplified using two pairs of primers: $5^{\prime}$-ctcgagCAGCTATTTAGTGAGGCTGTGAA-3', 5' -ggtaccG GAAGTGTCTTATCCGAGT-3' and 5 '-tctagaCAGC TATTTAGTGAGGCTGTGAA-3', 5' -ggatccGGAAGT GCTGTCTTATCCGAGT-3'. The resulting fragments were digested with Xho I, Kpn I and Xba I, BamH I, respectively, and inserted into the same sites of the pKANNIBAL vector. The resulting recombinant plasmid was then digested with Not $I$ and the fragment was inserted into pART27. All plasmids were verified by restriction digestion and DNA sequencing analysis.

The BnLACS2-OE and BnLACS2-RNAi constructs were introduced into the A. tumefaciens strain, GV3101, which were then used for the transformation. The transformation of canola plants was performed using the floral dip method [68]. Seeds were harvested from the transformed plant and screened for putative transformants. Positive BnLACS2-OE or -RNAi transgenic plants were first screened on selection medium containing hygromycin $(100 \mu \mathrm{g} / \mathrm{mL})$ or kanamycin $(50 \mu \mathrm{g} / \mathrm{mL})$, respectively, and then by $\mathrm{PCR}$ and RT-PCR using the primers presented in Additional file 5: Table S2).

\section{Determination of oil content and FAs in transgenic rapeseed}

Seed oil content was measured using near infrared spectrometry (NIRS) and nuclear magnetic resonance (NMR), as described in Bellincontro et al. [69]. The seeds (about $2.0 \mathrm{~g}$ ) of Zhongshuang 9 and independent $\mathrm{T}_{2}$ transgenic lines at the same growth stage were collected, and the oil contents were measured in triplicate. FA level was determined by GC-MS, as described above.

\section{Quantitative real-time PCR analysis}

The qRT-PCR of first-strand cDNA was performed using SYBR $^{\circ}$ PremixExTaq ${ }^{\text {TMII }}$ (TaKaRa, Japan), according to Wang et al. [70]. After optimizing the qRT-PCR conditions, the amplification efficiency was above $90 \%$ based on standard curves, Ct values ranged from 20.9 to 29.3, and all melt curves displayed a single specific peak. The relative expression of the target genes was normalized to the expression data of BnACTIN2 using the $2^{-\Delta \Delta C T}$ method [71]. All qRT-PCRs were performed in triplicate. The primers used for qRT-PCR analyses are listed in Additional file 6: Table S3.

\section{Supplementary information}

Supplementary information accompanies this paper at https://doi.org/10. $1186 /$ s12870-020-2240-x.

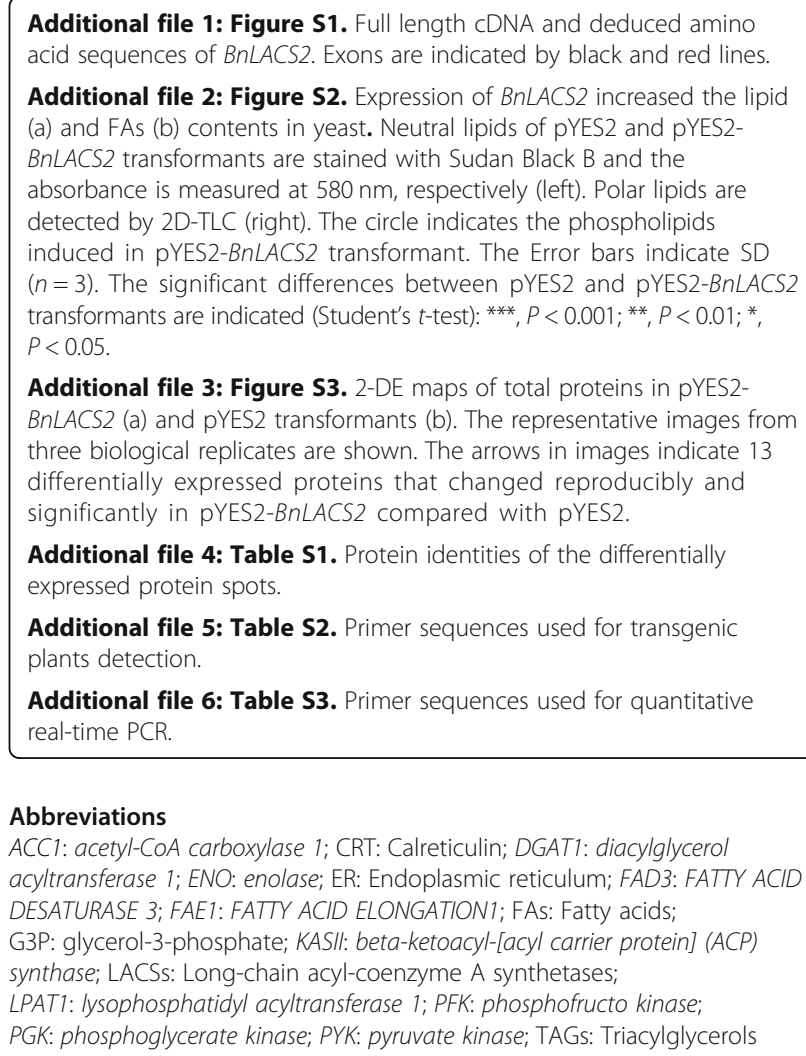

Additional file 3: Figure S3. 2-DE maps of total proteins in pYES2BnLACS2 (a) and pYES2 transformants (b). The representative images from three biological replicates are shown. The arrows in images indicate 13 differentially expressed proteins that changed reproducibly and significantly in pYES2-BnLACS2 compared with pYES2.

Additional file 4: Table S1. Protein identities of the differentially expressed protein spots.

Additional file 5: Table S2. Primer sequences used for transgenic plants detection.

Additional file 6: Table S3. Primer sequences used for quantitative real-time $P C R$.

\section{Abbreviations}

ACC1: acetyl-COA carboxylase 1; CRT: Calreticulin; DGAT1: diacylglycerol acyltransferase 1; ENO: enolase; ER: Endoplasmic reticulum; FAD3: FATTY ACID DESATURASE 3; FAE1: FATTY ACID ELONGATION1; FAs: Fatty acids; G3P: glycerol-3-phosphate; KASIl: beta-ketoacyl-[acyl carrier protein] (ACP) synthase; LACSs: Long-chain acyl-coenzyme A synthetases; LPAT1: lysophosphatidyl acyltransferase 1; PFK: phosphofructo kinase; PGK: phosphoglycerate kinase; PYK: pyruvate kinase; TAGs: Triacylglycerols

\section{Acknowledgements}

We thank Wen-Jie Chen of Hybid Rapeseed Research Center of Shannxi Province, for GC-MS analysis. We thank lan Graham and Tony Larson of Centre for Novel Agricultural Products (CNAP), Department of Biology University of York, for valuable advice on the experiment design.

\section{Authors' contributions}

X-LT conceived and designed research. S-LG, F-GZ Z-YM and L-ND conducted experiments. J-L, M-L and ZW contributed new reagents or analytical tools. $L$ ND and S-LG analyzed data. L-ND and X-LT wrote the manuscript. All authors read and approved the manuscript.

\section{Funding}

This work was supported by the National Key R \& D Program of China (2016YFD0101900 and 2016YFD0100305) and the National Natural Science 
Foundation of China (31471527 and 31271760). The funding agencies did not play any role in the design, analysis, or interpretation of this study and the relevant data.

\section{Availability of data and materials}

The datasets used and/or analyzed during the current study are available from corresponding authors on reasonable request.

\section{Ethics approval and consent to participate}

Not applicable.

\section{Consent for publication}

Not applicable.

\section{Competing interests}

The authors declare that they have no competing interests.

\section{Received: 21 March 2019 Accepted: 7 January 2020}

\section{Published online: 13 January 2020}

\section{References}

1. Jeong GT, Park DH. Batch (one- and two-stage) production of biodiesel fuel from rapeseed oil. Appl Biochem Biotechnol. 2006;131:668-79.

2. Kraljić K, Brkan V, Škevin D, Srček VG, Radošević K. Canolol dimer, a biologically active phenolic compound of edible rapeseed oil. Lipids. 2019;54:189-200.

3. Tan H, Yang X, Zhang F, Zheng X, Qu C, Mu J, et al. Enhanced seed oil production in canola by conditional expression of Brassica napus LEAFY COTYLEDON1 and LEC1-LIKE in developing seeds. Plant Physiol. 2011;156: 1577-88.

4. Liu J, Hao W, Liu J, Fan S, Zhao W, Deng L, et al. A novel chimeric mitochondrial gene confers cytoplasmic effects on seed oil content in polyploid rapeseed (Brassica napus). Mol Plant. 2019;12:582-6.

5. Cahoon EB, Shockey JM, Dietrich CR, Gidda SK, Mullen RT, Dyer JM. Engineering oilseeds for sustainable production of industrial and nutritional feedstocks: solving bottlenecks in fatty acid flux. Curr Opin Plant Biol. 2007; 10:236-44

6. Kelly AA, Quettier AL, Shaw E, Eastmond PJ. Seed storage oil mobilization is important but not essential for germination or seedling establishment in Arabidopsis. Plant Physiol. 2011;157:866-75.

7. Nasri N, Tlili N, Ben Ammar K, Khaldi A, Fady B, Triki S. High tocopherol and triacylglycerol contents in Pinus pinea L. seeds. Int J Food Sci Nutr. 2009;60: 161-9.

8. McFie PJ, Banman SL, Kary S, Stone SJ. Murine diacylglycerol acyltransferase2 (DGAT2) can catalyze triacylglycerol synthesis and promote lipid droplet formation independent of its localization to the endoplasmic reticulum. J Biol Chem. 2011;286:28235-46.

9. Groot PH, Scholte HR, Hülsmann WC. Fatty acid activation: specificity, localization, and function. Adv Lipid Res. 1976;14:75-126.

10. Hayashi H, De Bellis L, Hayashi Y, Nito K, Kato A, Hayashi M, et al. Molecular characterization of an Arabidopsis acyl-coenzyme a synthetase localized on glyoxysomal membranes. Plant Physiol. 2002;130:2019-26.

11. Soupene E, Kuypers FA. Mammalian long-chain acyl-CoA synthetases. Exp Biol Med (Maywood). 2008;233:507-21.

12. Fernandez RF, Kim SQ, Zhao Y, Foguth RM, Weera MM, Counihan JL, et al. Acyl-CoA synthetase 6 enriches the neuroprotective omega-3 fatty acid DHA in the brain. Proc Natl Acad Sci U S A. 2018;115:12525-30.

13. Shockey JM, Fulda MS, Browse JA. Arabidopsis contains nine long-chain acylcoenzyme a synthetase genes that participate in fatty acid and glycerolipid metabolism. Plant Physiol. 2002;129:1710-22

14. Gao F, Yang XF, Fu N, Hu Y, Ouyang Y, Qing K. Roles of long-chain acyl coenzyme a synthetase in absorption and transport of fatty acid. Chin Med Sci J. 2016:31:62-4

15. Li H, Melton EM, Quackenbush S, DiRusso CC, Black PN. Mechanistic studies of the long chain acyl-CoA synthetase Faalp from Saccharomyces cerevisiae. Biochim Biophys Acta. 1771;2007:1246-53.

16. Melton EM, Cerny RL, DiRusso CC, Black PN. Overexpression of human fatty acid transport protein 2/very long chain acyl-CoA synthetase 1 (FATP2/ Acsv/1) reveals distinct patterns of trafficking of exogenous fatty acids. Biochem Biophys Res Commun. 2013;440:743-8.
17. Guo X, Jiang M, Wan X, Hu C, Gong Y. Identification and biochemical characterization of five long-chain acyl-coenzyme a synthetases from the diatom Phaeodactylum tricornutum. Plant Physiol Biochem. 2014;74:33-41.

18. Jessen D, Roth C, Wiermer M, Fulda M. Two activities of long-chain acylcoenzyme a synthetase are involved in lipid trafficking between the endoplasmic reticulum and the plastid in Arabidopsis. Plant Physiol. 2015; 167:351-66.

19. Grevengoed TJ, Klett EL, Coleman RA. Acyl-CoA metabolism and partitioning. Annu Rev Nutr. 2014;34:1-30.

20. Zhang CL, Mao K, Zhou LJ, Wang GL, Zhang YL, Li YY, et al. Genome-wide identification and characterization of apple long-chain acyl-CoA synthetases and expression analysis under different stresses. Plant Physiol Biochem. 2018;132:320-32.

21. Schnurr JA, Shockey JM, de Boer GJ, Browse JA. Fatty acid export from the chloroplast. Molecular characterization of a major plastidial acyl-coenzyme a synthetase from Arabidopsis. Plant Physiol. 2002;129:1700-9.

22. Schnurr J, Shockey J, Browse J. The acyl-CoA synthetase encoded by LACS2 is essential for normal cuticle development in Arabidopsis. Plant Cell. 2004; 16:629-42.

23. Lü S, Song T, Kosma DK, Parsons EP, Rowland O, Jenks MA. Arabidopsis CER8 encodes LONG-CHAIN ACYL-COA SYNTHETASE 1 (LACS1) that has overlapping functions with LACS2 in plant wax and cutin synthesis. Plant J. 2009;59:553-64.

24. Weng H, Molina I, Shockey J, Browse J. Organ fusion and defective cuticle function in a lacs1 lacs2 double mutant of Arabidopsis. Planta. 2010;231: 1089-100.

25. Jessen D, Olbrich A, Knüfer J, Krüger A, Hoppert M, Polle A, et al. Combined activity of LACS1 and LACS4 is required for proper pollen coat formation in Arabidopsis. Plant J. 2011;68:715-26.

26. Fulda M, Schnurr J, Abbadi A, Heinz E, Browse J. Peroxisomal acyl-CoA synthetase activity is essential for seedling development in Arabidopsis thaliana. Plant Cell. 2004;16:394-405.

27. Zhao L, Katavic V, Li F, Haughn GW, Kunst L. Insertional mutant analysis reveals that long-chain acyl-CoA synthetase 1 (LACS1), but not LACS8, functionally overlaps with LACS9 in Arabidopsis seed oil biosynthesis. Plant J. 2010;64:1048-58.

28. Yu L, Tan X, Jiang B, Sun X, Gu S, Han T, et al. A peroxisomal long-chain acyl-CoA synthetase from Glycine max involved in lipid degradation. PLoS One. 2014;9:e100144

29. Aznar-Moreno JA, Venegas Calerón M, Martínez-Force E, Garcés R, Mullen R, Gidda SK, et al. Sunflower (Helianthus annuus) long-chain acyl-coenzyme a synthetases expressed at high levels in developing seeds. Physiol Plant. 2014:150:363-73.

30. Pongdontri P, Hills M. Characterization of a novel plant acyl-coA synthetase that is expressed in lipogenic tissues of Brassica napus L. Plant Mol Biol. 2001:47:717-26

31. Fulda M, Heinz E, Wolter FP. Brassica napus cDNAs encoding fatty acyl-CoA synthetase. Plant Mol Biol. 1997;33:911-22

32. Hung $\mathrm{CH}$, Kanehara $\mathrm{K}$, Nakamura $\mathrm{Y}$. In vivo reconstitution of algal triacylglycerol production in Saccharomyces cerevisiae. Front Microbiol. 2016;7:70

33. He X, Chen GQ Kang ST, McKeon TA. Ricinus communis contains an acylCoA synthetase that preferentially activates ricinoleate to its $\mathrm{COA}$ thioester. Lipids. 2007:42:931-8.

34. Li J, Zhao-Hui C, Batoux M, Nekrasov V, Roux M, Chinchilla D, et al. Specific ER quality control components required for biogenesis of the plant innate immune receptor EFR. Proc Natl Acad Sci U S A. 2009;106:15973-8.

35. Zubenko GS, Park FJ, Jones EW. Mutations in PEP4 locus of Saccharomyces cerevisiae block final step in maturation of two vacuolar hydrolases. Proc Natl Acad Sci U S A. 1983;80:510-4

36. Li R, Qiu Z, Wang X, Gong P, Xu Q, Yu QB, et al. Pooled CRISPR/Cas9 reveals redundant roles of plastidial phosphoglycerate kinases in carbon fixation and metabolism. Plant J. 2019:98:1078-89.

37. Schwender J, Ohlrogge JB, Shachar-Hill Y. A flux model of glycolysis and the oxidative pentosephosphate pathway in developing Brassica napus embryos. J Biol Chem. 2003;278:29442-53.

38. Andriotis VM, Kruger NJ, Pike MJ, Smith AM. Plastidial glycolysis in developing Arabidopsis embryos. New Phytol. 2010;185:649-62.

39. Baud S, Guyon V, Kronenberger J Wuillème $S$, Miquel $M$, et al. Multifunctional acetyl-CoA carboxylase 1 is essential for very long chain fatty acid elongation and embryo development in Arabidopsis. Plant J. 2003; 33:75-86. 
40. Yasuno R, von Wettstein-Knowles P, Wada H. Identification and molecular characterization of the beta-ketoacyl-[acyl carrier protein] synthase component of the Arabidopsis mitochondrial fatty acid synthase. J Biol Chem. 2004:279:8242-51.

41. Chiron H, Wilmer J, Lucas MO, Nesi N, Delseny M, Devic M, et al. Regulation of FATTY ACID ELONGATION1 expression in embryonic and vascular tissues of Brassica napus. Plant Mol Biol. 2015;88:65-83.

42. Lou Y, Schwender J, Shanklin J. FAD2 and FAD3 desaturases form heterodimers that facilitate metabolic channeling in vivo. J Biol Chem. 2014; 289:17996-8007.

43. Misra N, Panda PK, Parida BK. Genome-wide identification and evolutionary analysis of algal LPAT genes involved in TAG biosynthesis using bioinformatic approaches. Mol Biol Rep. 2014;41:8319-32.

44. Caldo KM, Greer MS, Chen G, Lemieux MJ, Weselake RJ. Purification and properties of recombinant Brassica napus diacylglycerol acyltransferase 1. FEBS Lett. 2015;589:773-8.

45. Ichihara K, Kobayashi N, Saito K. Lipid synthesis and acyl-CoA synthetase in developing rice seeds. Lipids. 2003;38:881-4.

46. Babbitt PC, Kenyon GL, Martin BM, Charest $H$, Slyvestre M, Scholten JD, et al. Ancestry of the 4-chlorobenzoate dehalogenase: analysis of amino acid sequence identities among families of acyl:adenyl ligases, enoyl-CoA hydratases/isomerases, and acyl-CoA thioesterases. Biochemistry. 1992;31: 5594-604.

47. Wang XL, Li XB. The GhACS1 gene encodes an acyl-CoA synthetase which is essential for normal microsporogenesis in early anther development of cotton. Plant J. 2009;57:473-86

48. Xu Y, Holic R, Li D, Pan X, Mietkiewska E, Chen G, et al. Substrate preferences of long-chain acyl-CoA synthetase and diacylglycerol acyltransferase contribute to enrichment of flax seed oil with a-linolenic acid. Biochem J. 2018:475:1473-89.

49. Tan XL, Huang Q, Tan RK, Wu L, Li XF. Cloning and functional characterization of a fatty acyl-acyl carrier protein thioesterase gene (BnFatB) in Brassica napus L. J Agric Sci Tech. 2015;17:987-97.

50. Avidan O, Brandis A, Rogachev I, Pick U. Enhanced acetyl-CoA production is associated with increased triglyceride accumulation in the green alga Chlorella desiccata. J Exp Bot. 2015;66:3725-35.

51. Kerner J, Minkler PE, Lesnefsky EJ, Hoppel CL. Fatty acid chain elongation in palmitate-perfused working rat heart: mitochondrial acetyl-CoA is the source of two-carbon units for chain elongation. J Biol Chem. 2014;289: 10223-34.

52. Igal RA, Wang P, Coleman RA. Triacsin C blocks de novo synthesis of glycerolipids and cholesterol esters but not recycling of fatty acid into phospholipid: evidence for functionally separate pools of acyl-CoA. Biochem J. 1997;324:529-34

53. Higgins DG, Thompson JD, Gibson TJ. Using CLUSTAL for multiple sequence alignments. Methods Enzymol. 1996;266:383-402.

54. Tamura K, Peterson D, Peterson N, Stecher G, Nei M, Kumar S. MEGA5: molecular evolutionary genetics analysis using maximum likelihood, evolutionary distance, and maximum parsimony methods. Mol Biol Evol. 2011;28:2731-9.

55. Karimi M, Inze D, Depicker A. GATEWAY vectors for Agrobacterium-mediated plant transformation. Trends Plant Sci. 2002;7:193-5.

56. Wood CC, Petrie JR, Shrestha P, Mansour MP, Nichols PD, Green AG, et al. A leaf-based assay using interchangeable design principles to rapidly assemble multistep recombinant pathways. Plant Biotechnol J. 2009;7:914-24.

57. Chen S, Songkumarn P, Liu J, Wang GL. A versatile zero background Tvector system for gene cloning and functional genomics. Plant Physiol. 2009;150:1111-21.

58. Gietz RD, Schiestl RH. Frozen competent yeast cells that can be transformed with high efficiency using the LiAc/SS carrier DNA/PEG method. Nat Protoc. 2007:2:1-4.

59. Thakur MS, Prapulla SG, Karanth NG. Estimation of intracellular lipids by the measurement of absorbance of yeast cells stained with Sudan Black B. Enzym Microb Technol. 1989;11:252-4.

60. Bligh EG, Dyer WJ. A rapid method of total lipid extraction and purification. Can J Biochem Physiol. 1959;37:911-7.

61. Guan Z, Tian B, Perfumo A, Goldfine H. The polar lipids of Clostridium psychrophilum, an anaerobic psychrophile. Biochim Biophys Acta. 1831; 2013:1108-12.
62. Brandenburg J, Blomqvist J, Pickova J, Bonturi N, Sandgren M, Passoth V. Lipid production from hemicellulose with Lipomyces starkeyi in a $\mathrm{pH}$ regulated fed-batch cultivation. Yeast. 2016;33:451-62.

63. Mu J, Tan H, Zheng Q, Fu F, Liang Y, Zhang J, et al. LEAFY COTYLEDON1 is a key regulator of fatty acid biosynthesis in Arabidopsis. Plant Physiol. 2008; 148:1042-54.

64. Isola D, Marzban G, Selbmann L, Onofri S, Laimer M, Sterflinger K. Sample preparation and 2-DE procedure for protein expression profiling of black microcolonial fungi. Fungal Biol. 2011;115:971-7.

65. Bradford MM. A rapid and sensitive method for the quantification of microgram quantities of protein utilizing the principle of protein-dye binding. Anal Biochem. 1976;72:248-54.

66. Ding LN, Yang RY, Yang GX, Cao J, Li P, Zhou Y. Identification of putative phosphoproteins in wheat spikes induced by Fusarium graminearum. Planta. 2016:243:719-31.

67. Ding LN, Li M, Li P, Cao J. Comparative proteomics analysis of young spikes of wheat in response to Fusarium graminearum infection. Acta Physiol Plant. 2017;39:271.

68. Clough SJ, Bent AF. Floral dip: a simplified method for agrobacteriummediated transformation of Arabidopsis thaliana. Plant J. 1998;16:735-43.

69. Bellincontro A, Caruso G, Mencarelli F, Gucci R. Oil accumulation in intact olive fruits measured by near infrared spectroscopy-acousto-optically tunable filter. J Sci Food Agric. 2013;93:1259-65.

70. Wang YK, Li YL, Fu ZL, Huang Q, Yue XG, Wang Y, et al. Transcriptome analysis of Brassica napus wax-deficient mutant revealed the dynamic regulation of leaf wax biosynthesis is associated with basic pentacysteine 6 . Int J Agric Biol. 2019;21:1228-34.

71. Livak KJ, Schmittgen TD. Analysis of relative gene expression data using realtime quantitative PCR and the $2^{-\Delta \Delta C T} T$ method. Methods. 2001;25:402-8.

\section{Publisher's Note}

Springer Nature remains neutral with regard to jurisdictional claims in published maps and institutional affiliations.

\section{Ready to submit your research? Choose BMC and benefit from:}

- fast, convenient online submission

- thorough peer review by experienced researchers in your field

- rapid publication on acceptance

- support for research data, including large and complex data types

- gold Open Access which fosters wider collaboration and increased citations

- maximum visibility for your research: over $100 \mathrm{M}$ website views per year

At BMC, research is always in progress.

Learn more biomedcentral.com/submissions 